\title{
Quantum Annealing Simulation of Out-of-Equilibrium Magnetization in a Spin-Chain Compound
}

\author{
Andrew D. King $\odot,{ }^{1,}{ }^{*}$ Cristian D. Batista $\odot,{ }^{2,3}$ Jack Raymond $\odot,{ }^{1}$ Trevor Lanting $\odot,{ }^{1}$ Isil Ozfidan, ${ }^{1}$ \\ Gabriel Poulin-Lamarre $\odot,{ }^{1}$ Hao Zhang $\odot,{ }^{2,4}$ and Mohammad H. Amin ${ }^{1,5}$ \\ ${ }^{1}$ D-Wave, Burnaby, British Columbia V5G 4M9, Canada \\ ${ }^{2}$ Department of Physics and Astronomy, University of Tennessee, Knoxville, Tennessee 37996-1200, USA \\ ${ }^{3}$ Neutron Scattering Division and Shull-Wollan Center, Oak Ridge National Laboratory, Oak Ridge, \\ Tennessee 37831, USA \\ ${ }^{4}$ Materials Science and Technology Division, Oak Ridge National Laboratory, Oak Ridge, Tennessee 37831, USA \\ ${ }^{5}$ Department of Physics, Simon Fraser University, Burnaby, British Columbia V5A 1S6, Canada
}

(Received 12 January 2021; revised 28 June 2021; accepted 12 July 2021; published 30 July 2021)

\begin{abstract}
Geometrically frustrated spin-chain compounds such as $\mathrm{Ca}_{3} \mathrm{Co}_{2} \mathrm{O}_{6}$ exhibit extremely slow relaxation under a changing magnetic field. Consequently, both low-temperature laboratory experiments and Monte Carlo simulations have shown peculiar out-of-equilibrium magnetization curves, which arise from trapping in metastable configurations. In this work, we simulate this phenomenon in a superconducting quantum annealing processor, allowing us to probe the impact of quantum fluctuations on both the equilibrium and dynamics of the system. Increasing the quantum fluctuations with a transverse field reduces the impact of metastable traps in out-of-equilibrium samples and aids the development of three-sublattice ferrimagnetic (up-up-down) long-range order with magnetization 1/3. At equilibrium, we identify a finitetemperature shoulder in the 1/3-to-saturated phase transition, promoted by quantum fluctuations but with an entropic origin. This work demonstrates the viability of dynamical as well as equilibrium studies of frustrated magnetism using large-scale programmable quantum systems and is therefore an important step toward programmable simulation of dynamics in materials using quantum hardware.
\end{abstract}

DOI: 10.1103/PRXQuantum.2.030317

\section{INTRODUCTION}

Geometrically frustrated magnetic systems exhibit a variety of interesting dynamical and equilibrium properties. The calcium oxalate $\mathrm{Ca}_{3} \mathrm{Co}_{2} \mathrm{O}_{6}$, a canonical example of a geometrically frustrated spin-chain compound, can be extremely slow to relax from metastable configurations when subjected to a changing magnetic field. The magnetic $\mathrm{Co}^{3+}$ ions align in ferromagnetic (FM) spin chains along the $c$ axis that form a triangular antiferromagnetic lattice in the $a-b$ plane. This compound shows unusual out-of-equilibrium magnetization curves, a phenomenon that has been studied both in situ [1-5] and in simulation with a variety of simulated two-dimensional (2D) and three-dimensional (3D) models [6-9]. Hysteresis in this system shows a counterintuitive response to changes in

\footnotetext{
*aking@dwavesys.com
}

Published by the American Physical Society under the terms of the Creative Commons Attribution 4.0 International license. Further distribution of this work must maintain attribution to the author(s) and the published article's title, journal citation, and DOI. a longitudinal field: increasing the field sweep rate can increase the response to the changing field, as measured by bulk magnetization. Simulations implicate the FM spin chains in the slow dynamics and indicate the importance of intrachain physics in correctly understanding the magnetic behavior of $\mathrm{Ca}_{3} \mathrm{Co}_{2} \mathrm{O}_{6}$ as a rare example of effective lowdimensional Ising-like triangular frustration. Some models have suggested quantum tunneling within the FM spin chains [10]; the influence of quantum fluctuations on relaxation in spin-chain compounds is therefore an important question.

Two decades ago, Brooke et al. explored the role of quantum fluctuations for the disordered spin glass $\mathrm{LiHo}_{x} \mathrm{Y}_{1-x} \mathrm{~F}_{4}$, annealing it to a low-energy state in a laboratory by attenuating thermal and quantum fluctuations [11]. Their finding that quantum annealing relaxed the system faster than thermal annealing is a major motivating factor in the development of programmable superconducting quantum annealing (QA) processors [12]. In turn, recent experiments have demonstrated that a class of quantum condensed-matter systems can be simulated using QA processors [13-17]. One such system is a geometrically frustrated 2D Ising magnet (Fig. 1), in which 


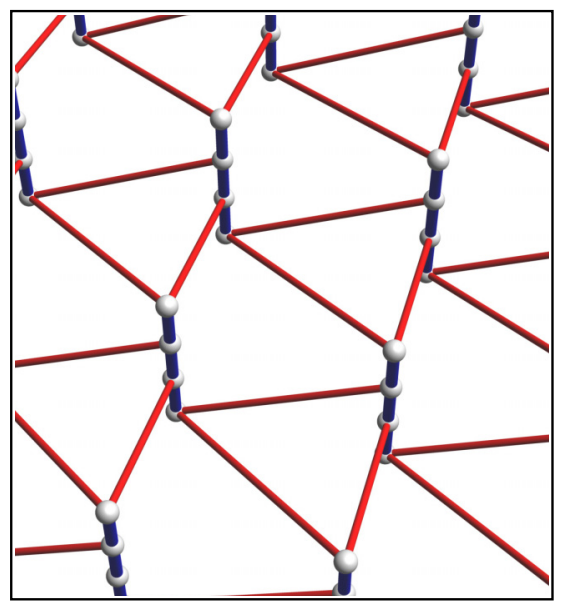

FIG. 1. A geometrically frustrated square-octagonal lattice viewed as a spin-chain antiferromagnet. The red and blue lines indicate antiferromagnetic and ferromagnetic Ising exchange with couplings $J_{1}$ and $J_{0}=-1.8 J_{1}$, respectively, where ferromagnetic bonds form four-spin chains. As in $\mathrm{Ca}_{3} \mathrm{Co}_{2} \mathrm{O}_{6}$, the strong FM chains interact via weaker AFM couplings in a triangular geometry.

quantum fluctuations induce order by disorder (OBD) at zero longitudinal field. Upon increasing temperature, this ordering is suppressed via a sequence of two BerezinskiiKosterlitz-Thouless (BKT) transitions, a phenomenon previously demonstrated using path-integral quantum Monte Carlo (QMC) simulations [18] and later using QA simulation to probe equilibrium and dynamical properties $[14,15]$.

In this work, we use a quantum annealing processor to simulate a spin-chain antiferromagnet and study the effects of tuning the quantum fluctuations on both dynamical and equilibrium properties. In the next section, we present the simulated spin-chain antiferromagnet in the transverse field Ising model, and discuss its relationship to real magnetic compounds. In Sec. III, we study equilibrium properties, confirming agreement between our quantum annealing experiments and the thermodynamic phase diagram and, in particular, noting a shoulder in the magnetization curve caused by the transverse field. In Sec. IV, we study hysteresis in the system, reproducing the out-of-equilibrium metastability seen in previous classical simulations and laboratory experiments.

\section{SPIN-CHAIN MODEL AND RELATED COMPOUNDS}

Here, we consider the lattice studied in Refs. [14,15] as a spin-chain antiferromagnet (Fig. 1) and simulate it under a longitudinal magnetic field, in addition to the transverse field that induces quantum fluctuations. The Hamiltonian of the system is

$$
\begin{aligned}
\mathcal{H}= & B \sum_{j ; v \in\{1,2,3,4\}} \sigma_{j v}^{z}+J_{1} \sum_{\langle j, l\rangle} \sigma_{j v(j, l)}^{z} \sigma_{l v(l, j)}^{z} \\
& -J_{0} \sum_{j ; v \in\{1,2,3\}} \sigma_{j v}^{z} \sigma_{j v+1}^{z}-\Gamma \sum_{j ; v \in\{1,2,3,4\}} \sigma_{j v}^{x},
\end{aligned}
$$

where $v \in\{1,2,3,4\}$ is the spin index of each vertical chain and $v(j, l)$ is the spin index of the chain $j$ that interacts with a spin in the chain $l$. The intrachain interaction is ferromagnetic $\left(J_{0}>0\right)$ and the interchain interaction is antiferromagnetic $\left(J_{1}>0\right)$. The Hamiltonian parameters can be expressed as $B(t)=\mathcal{J}(t) H(t), J_{0}(t)=\mathcal{J}(t) I_{0}$, $J_{1}(t)=\mathcal{J}(t) I_{1}$, and $\Gamma(t)$ to indicate the time-dependent control over strengths of the Ising, longitudinal field, and transverse field terms, respectively, where $I_{0}=1.8, I_{1}=1$ and $\mathcal{J}(t) \geq 0$. Note that the ratio between the FM and the AFM Ising interactions is time-independent $J_{0} / J_{1}=$ $I_{0} / I_{1}=1$.8. $H(t)$ ranges from 0 to 2 . Thus we can alternatively express the time-dependent Hamiltonian as

$$
\begin{aligned}
\mathcal{H}= & \mathcal{J}(t)\left(H(t) \sum_{j ; v \in\{1,2,3,4\}} \sigma_{j v}^{z}+\sum_{\langle j, l\rangle} \sigma_{j v(j, l)}^{z} \sigma_{l v(l, j)}^{z}\right. \\
& \left.-1.8 \sum_{j ; v \in\{1,2,3\}} \sigma_{j v}^{z} \sigma_{j v+1}^{z}\right)-\Gamma(t) \sum_{j ; v \in\{1,2,3,4\}} \sigma_{j v}^{x}
\end{aligned}
$$

where $\mathcal{J}(t)$ and $\Gamma(t)$ are the time-dependent Ising and transverse energy functions comprising the annealing schedule, familiar in the field of quantum annealing [13]. The choices of $I_{0}=1.8$ and $I_{1}=1$ ensure that - as in related materials of interest - spin chains have few breaks at equilibrium and the lattice can be fully saturated with $H=2$.

While the spin Hamiltonian $\mathcal{H}$ cannot be directly applied to any real material, it can still be used to model the behavior of known quantum magnets. In addition to $\mathrm{Ca}_{3} \mathrm{Co}_{2} \mathrm{O}_{6}$ [19-23], the compound $\mathrm{TmMgGaO}$ has recently become the subject of intense study [24-28]. Here, the magnetic $\mathrm{Tm}^{3+}$ ions form a perfect triangular lattice. These moments can be described in terms of an effective spin- $1 / 2$ variable because the two lowest crystal-field (CF) levels are separated from the rest by an energy gap that is much bigger than the Ising-like exchange interaction between different magnetic moments. Given that the $\mathrm{Tm}^{3+}$ ion has total angular momentum $J=6$, there is no Kramers degeneracy and the two lowest energy CF levels are singlets [26,27]. The energy splitting between the two singlets corresponds to an intrinsic transverse field acting on the spin- $1 / 2$ variables and the low-energy physics of $\mathrm{Tm}^{3+}$ is described by a transverse field Ising model (TFIM) on a triangular lattice [26,27,29], which coincides with the effective low-energy model for $\mathcal{H}$ in the limit 
$\Gamma / J_{0} \ll 1$. In this limit, the low-energy degrees of freedom of each chain are also spin-1/2 variables that interact via the effective Hamiltonian

$$
\tilde{\mathcal{H}}=\tilde{B} \sum_{j} \tilde{\sigma}_{j}^{z}+\tilde{J}_{1} \sum_{\langle j, l\rangle} \tilde{\sigma}_{j}^{z} \tilde{\sigma}_{l}^{z}-\tilde{\Gamma} \sum_{j} \tilde{\sigma}_{j}^{x},
$$

where $\tilde{B}=4 B, \tilde{J}_{1}=J_{1}$ and $\tilde{\Gamma}=\Gamma^{4} / J_{0}^{3}$.

\section{EQUILIBRIUM PROPERTIES}

As in Ref. [14], we program the QA processor to realize a cylindrical lattice on 1800 spins; in this experiment, defects reduce the number of working spins to 1764 . To suppress boundary effects and better simulate the thermodynamic limit, we tune the longitudinal field and coupling terms on a per-device basis to empirically homogenize qubit magnetizations and coupled spin-spin correlations, respectively [14,30,31]. This approach additionally suppresses the effect of site vacancies caused by inoperable qubits - six of 450 sites are vacant in the quantum annealing experiments.

We estimate the statistics of the system at thermal equilibrium under a fixed longitudinal magnetic field $B(t)=$ $B(0)$ that ranges from 0 to 2 , using the "quantum evolution Monte Carlo" (QEMC) method introduced in Ref. [14]. In this mode of operation, the system is initialized in a random classical spin state and repeatedly exposed to both quantum and thermal fluctuations, in this case for $500 \mu$ s per exposure. Between exposures, the fluctuations are quenched as the system is destructively projected to the computational $\sigma^{z}$ basis and a classical state is read (see Appendix A).

Because the Ising system is repeatedly quenched and relaxed, the observed output does not precisely reflect the system at equilibrium. Rather, prior to each readout, the quantum and thermal fluctuations are turned off and a small amount of local relaxation occurs during this process - most obviously in the erasure of single-spin excitations. Bulk observables reflecting long-range order or magnetization are relatively protected from this distortion [15]. One can therefore think of the equilibrium estimates in two ways: first, as a perturbed observation of the system at equilibrium or, second, as an observation of a periodically driven Floquet system.

Figure 2 shows the thermodynamic phase diagram obtained with classical $(\Gamma=0)$ and quantum $(\Gamma \neq 0)$ Monte Carlo and the transitions that are extracted from the QA processor for the QEMC runs. In the classical Ising limit $(\Gamma=0)$, the phase diagram only includes two phases. The ordered phase at low enough temperature and longitudinal field $B \neq 0$ corresponds to a threefold degenerate three-sublattice up-up-down ferrimagnetic (FIM) state that induces a $1 / 3$ plateau in the magnetization curve $M(B) / M_{\text {sat }}\left(M=\left\langle\sum_{j v} \sigma_{j v}^{z}\right\rangle\right)$ and can be characterized by

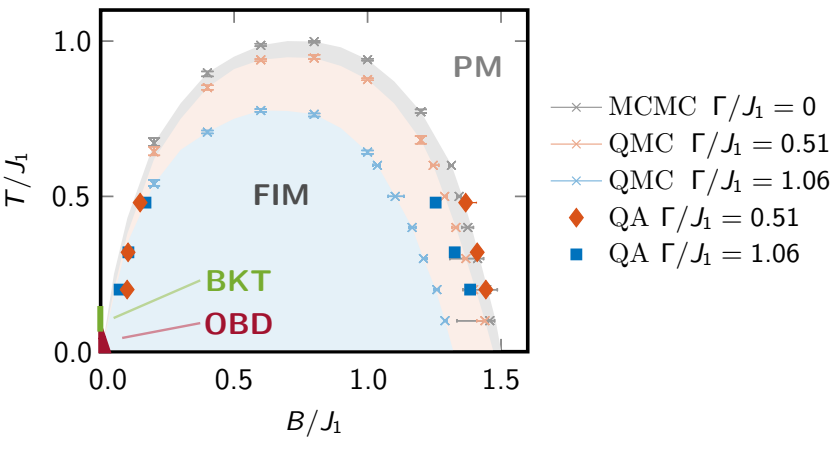

FIG. 2. The thermodynamic phase diagram of $\mathcal{H}$ for different transverse fields $\Gamma$. For fixed value of $\Gamma / J_{1}$, the system has a FIM phase separated by a PM phase; the phase-transition locations are estimated using Monte Carlo and QA simulations. For $\Gamma>0$, $B=0$, there are two BKT transitions delimiting a critical phase (BKT) and an OBD phase [14,27,29].

the complex order parameter

$$
\psi=m e^{i \theta}=\left(m_{1}+m_{2} e^{2 \pi i / 3}+m_{3} e^{4 \pi i / 3}\right) / \sqrt{3},
$$

where $m_{1}, m_{2}$, and $m_{3}$ are individual sublattice magnetizations [18]. The three ground states of the $1 / 3$ plateau have $\psi$ equal to $-(2 / \sqrt{3}) e^{k 2 \pi i / 3}$ for $k=0,1,2$. Thus to measure the onset of FIM long-range order (LRO) in our simulations of finite size lattices, we use the normalized cubic invariant

$$
m_{\mathrm{FIM}}=-\operatorname{Re}\left[(\psi \sqrt{3} / 2)^{3}\right]=-m^{3} \cos (3 \theta)(\sqrt{3} / 2)^{3} .
$$

The second phase is simply the paramagnetic (PM) state that becomes a fully polarized state along the longitudinal field direction at $T=0$.

As shown in Fig. 2, the FIM phase survives for $\Gamma \neq 0$. In addition, there are two low-temperature phases identified in the $B=0$ case: a critical phase and an ordered phase, in which $\psi$ concentrates around six values rather than three [32]. This is also a three-sublattice ordering in which one of the two spin-up sublattices of the FIM state is polarized along the $x$ direction. The critical phase disappears for finite $B$ with the two BKT transitions that mark its upper and lower boundary. For $B \ll \Gamma$, the system transitions directly from the PM state to a sixfold degenerate state upon cooling, with the spins of the third sublattice canted along the longitudinal field direction. Unfortunately, the base temperature of the QA processor is not low enough to access this ordered phase [14].

The phase-transition points between the FIM and the PM phases can be determined with the QA processor (blue and red squares in Fig. 2) by measuring the crossing point of $m_{\mathrm{FIM}}=1 / 2$. The transition is accompanied by a rather abrupt change of the magnetization that produces a peak in the longitudinal susceptibility $d M / d B$. As shown in Fig. 3, this peak splits into two upon increasing the temperature 


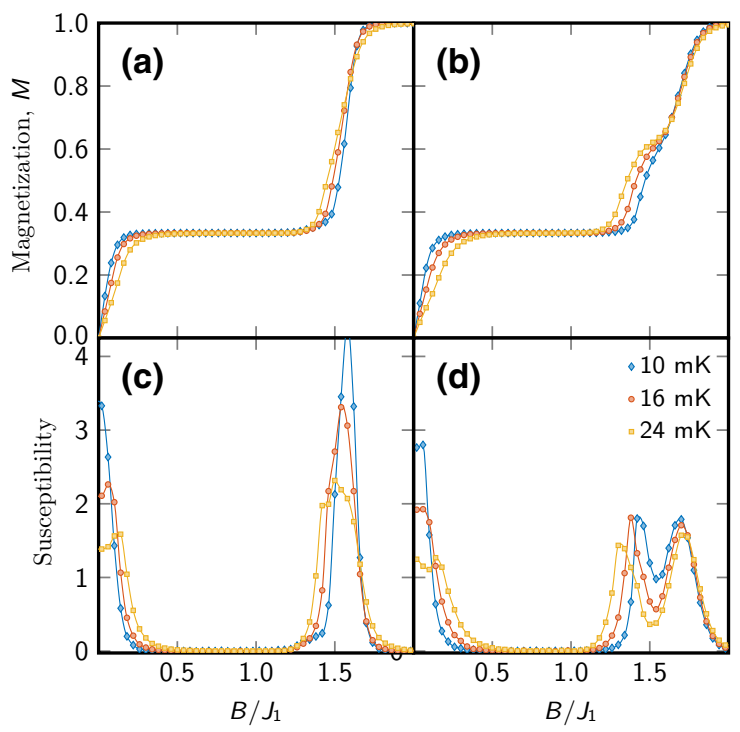

FIG. 3. Equilibrium magnetization and susceptibility observations: (a),(c) low tunneling, $\Gamma / J_{1}=0.51$; (b),(d) high tunneling, $\Gamma / J_{1}=1.06$. The susceptibility is computed as $d M / d\left(B / B_{\mathrm{MAX}}\right)$. In both cases, $J_{1} \approx 46 \mathrm{mK}$. Increasing temperature corresponds to broadening of the transverse-field-induced magnetization shoulder, consistent with an entropic origin.

and the transverse field. The lower field peak still signals the metamagnetic phase transition between the FIM and the PM phases. The higher field peak results from the combined effect of quantum and thermal fluctuations. As such, this second peak in $d M / d B$, or the corresponding magnetization shoulder in $M(B)$ (see Fig. 3), can be used as an experimental fingerprint of the presence of a transverse field in a 2D Ising system. In other words, the doublepeak structure can be used to identify materials that are realizations of 2D quantum Ising models.

To understand the origin of the second peak, it is convenient to start from the $\tilde{\Gamma}=0$ limit of $\tilde{\mathcal{H}}$. In this classical limit, $\tilde{\mathcal{H}}$ has an extensive ground-state degeneracy at the effective saturation field $\tilde{B}=\tilde{B}_{\text {sat }}=4 B_{\text {sat }}=6 J_{1}$. At this value of the field, the energy cost of flipping a spin is equal to zero as long as the neighboring spins are polarized along the field direction. Since the fully polarized state is a ground state at $B=\tilde{B}_{\text {sat }}$, the ground space $\mathcal{S}$ of $\tilde{\mathcal{H}}\left(\tilde{\Gamma}=0, \tilde{B}=\tilde{B}_{\text {sat }}\right)$ is generated by spin states $\left|\phi_{j}\right\rangle$ that do not contain any pair of nearest-neighbor spins antialigned with the longitudinal field $B$. The extensive ground-state degeneracy is lifted by the transverse field. To first order in $\tilde{\Gamma}$, the new ground state is obtained by diagonalizing the $\tilde{\Gamma}$ term restricted to $\mathcal{S}$. Since the $\tilde{\Gamma}$ term is irreducible and nonpositive on $\mathcal{S}$, the Perron-Frobenius theorem guarantees that the transverse field removes the ground-state degeneracy completely. This observation implies that the new ground state at $\tilde{B}=6 J_{1}$ is a quantum paramagnet corresponding to a linear superposition of all $\left|\phi_{j}\right\rangle$. This result implies that the new critical field for the FIM-PM transition must be lower than $\tilde{B}_{\text {sat }}=6 J_{1}: \tilde{B}_{c}(\tilde{\Gamma}, T=0)=6 J_{1}-\delta \tilde{B}$ with $\delta \tilde{B} \simeq \tilde{\Gamma}$. This estimate results from requiring that the sum of the molecular and the applied field, $6 J_{1}-\tilde{B}_{c}$ acting on an effective spin down of the FIM state must be comparable to the energy gain per site (of order $\tilde{\Gamma}$ ) of the quantum paramagent. As shown in Fig. 3(a), the discontinuous change of $M$ at $B=B_{\text {sat }}$ and $\Gamma=0$ is replaced by a linear magnetization ramp that connects the $1 / 3$ plateau $\left(M / M_{\text {sat }}=1 / 3\right)$ with the saturated value $M=M_{\text {sat }}$. The slope of this ramp is approximately $\left(1 / M_{\text {sat }}\right) \delta M / \delta \tilde{B} \simeq$ $1 / 3 \tilde{\Gamma}$.

Since the stabilization energy of the quantum paramagnetic state at $\tilde{B}=6 J_{1}$ is of order $\tilde{\Gamma}$, the extensive ground-state entropy must reappear at a finite temperature $T \simeq \tilde{\Gamma}$ and control the equilibrium properties. This observation implies that at this temperature, the magnetization should take the value $M_{m} / M_{\text {sat }}$ that is supported by the maximum number of states $\left|\phi_{j}\right\rangle$, i.e., the value that maximizes the entropy within the low-energy sector $\mathcal{S}$. A simple numerical simulation, which is described in Appendix B, gives $M_{m} / M_{\text {sat }} \simeq 0.575$ in the thermodynamic limit and $M_{m} / M_{\text {sat }} \simeq 0.58$ for the lattices sizes considered in this work. We note that this value is in good agreement with the onset of the magnetization shoulder near the middle of the magnetization ramp, which is shown in Fig. 3(b). Further evidence for the entropic origin of the shoulder is also provided in Appendix B. According to this entropy-based argument, the shoulder should develop at $T \simeq \tilde{\Gamma}$ and the width of the shoulder should be of order $\tilde{\Gamma}$ (note that $\delta \tilde{B} \simeq \tilde{\Gamma}$ ). In other words, the shoulder is the combined effect of quantum fluctuations that stabilize the low-energy manifold $\mathcal{S}$ over a finite longitudinal field interval of order $\Gamma$ and thermal fluctuations that select the submanifold of states with magnetization $M \simeq M_{m}$. We note that an intermediate regime between the FIM and the fully polarized phases has been detected with neutronscattering measurements of $\mathrm{TmMgGaO}_{4}$ [28]. However, it is difficult to separate the relative roles of the intrinsic quantum and thermal fluctuations of the clean limit versus the spatial fluctuations induced by the significant amount of disorder present in $\mathrm{TmMgGaO}_{4}$.

$\mathrm{Ca}_{3} \mathrm{Co}_{2} \mathrm{O}_{6}$ comprises a triangular lattice of ferromagnetic (FM) Ising chains coupled by weak antiferromagnetic (AFM) exchange interactions. This compound exhibits longitudinal field-induced magnetization steps, the height of which depends on the field sweep history and rate [1923]. These out-of-equilibrium magnetization steps, which appear at regular magnetic field intervals, originate from the extensive ground-state degeneracy of the triangular Ising model and by the need to overcome an energy barrier to connect different ground states by a sequence of individual spin flips (local dynamics). As has been pointed out in Ref. [33], the regular spacing between the magnetization steps can be explained by the regular spacing between the 
molecular fields producing the six nearest-neighbor spins that surround the spin to be flipped. Experimental studies of the quantum effect of an external transverse field in $\mathrm{Ca}_{3} \mathrm{Co}_{2} \mathrm{O}_{6}$ are challenging because of the very small effective gyromagnetic factor in the direction perpendicular to the chains.

\section{OUT-OF-EQUILIBRIUM BEHAVIOR}

Having described simulations of the system at equilibrium, we move on to the case of a varying longitudinal field, using the same lattice and the same methods for approximating the large-system limit as used in the previous section (cf. Ref. [30,31]).

We simulate relaxation of the system under field $B(t)$ that either increases from $B(0)=0$ or decreases from a saturating field $B(0)=2 J_{1}$. Figure $4(\mathrm{a})$ shows the timedependent Hamiltonian terms for a $100 \mu$ s field sweep, which follows a $100-\mu$ s anneal to the desired values of $\Gamma$ and $J_{1}$ (cf. Ref. [13], Fig. S13). Since QA readout is achieved after projection to the $\sigma^{z}$ basis following a rapid quench of both $\Gamma$ and $J_{1}$, each value of $B / J_{1}$ must be simulated individually, as opposed to taking multiple measurements from a single sweep of $B$. We probe sweep rates of $1 \mu \mathrm{s}, 10 \mu \mathrm{s}$, and $100 \mu \mathrm{s}$ for a low and high transverse field $\left(\Gamma / J_{1}=0.51\right.$ and 1.06$) ; J_{1}=1.89 \mathrm{GHz}$ is roughly 4.5 times the effective qubit temperature $T=10 \mathrm{mK}$. As the sweep of $B$ becomes slower, the observations approach equilibrium values, which we estimate using a QEMC protocol in which the system is relaxed iteratively [14], in contrast to the single-shot measurements.

Figure 4(b) shows magnetization hysteresis curves for different sweep rates. As in investigations of $\mathrm{Ca}_{3} \mathrm{Co}_{2} \mathrm{O}_{6}$, we observe distinct out-of-equilibrium behavior, including metastable overshooting of the equilibrium $1 / 3$ plateau. Increasing the transverse field $\Gamma / J_{1}$ from 0.51 to 1.06 significantly reduces the signatures of metastability in the magnetization curves.

The $1 \mu$ s sweep of $B$ results in almost no LRO when $\Gamma / J_{1}=0.51$ despite the order seen at thermal equilibrium [Fig. 4(c)]. Throughout the curve, increasing $\Gamma / J_{1}$ to 1.06 appears to hasten the development of LRO by over an order of magnitude. In this sense, it is clear that quantum fluctuations suppress metastable trapping in the out-ofequilibrium experiment. As in the case of $\mathrm{Ca}_{3} \mathrm{Co}_{2} \mathrm{O}_{6}$ [34], the out-of-equilibrium simulation of a $1-\mu$ s sweep also shows three steps, in addition to the above-mentioned shoulder. It is important to note that $\mathcal{H}$ is far from being a realistic model for $\mathrm{Ca}_{3} \mathrm{Co}_{2} \mathrm{O}_{6}$. Besides the fact that $\mathrm{Ca}_{3} \mathrm{Co}_{2} \mathrm{O}_{6}$ is a $3 \mathrm{D}$ material, a more realistic Hamiltonian should include multiple competing interchain exchange interactions that induce a commensurate-incommensurate transition at finite temperature [6]. Nevertheless, as has been shown by Kudasov [33], a simplified 2D triangular Ising model can explain the observation of three outof-equilibrium steps, so it is not surprising that a time
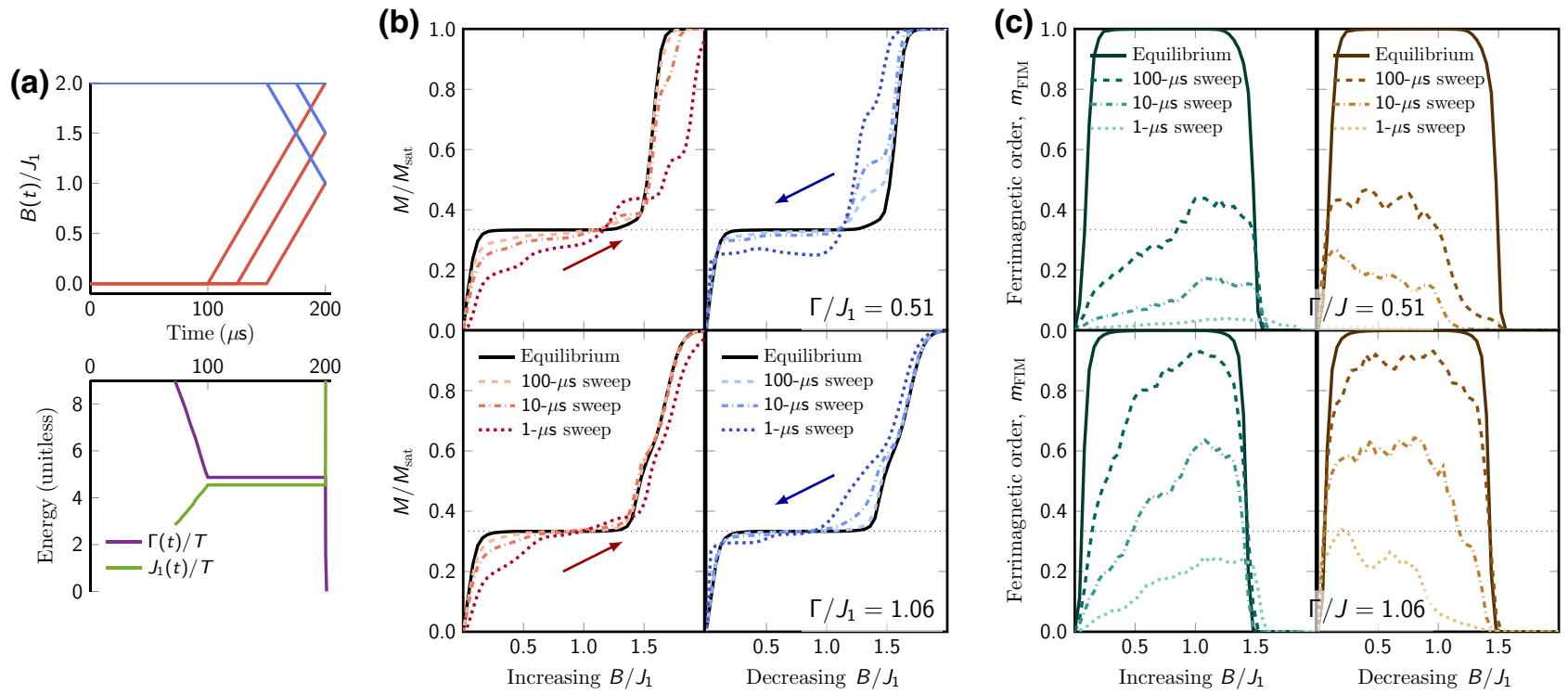

FIG. 4. Out-of-equilibrium experimental results obtained from hysteresis simulation. (a) Annealing protocols for simulating hysteresis. To take an observation at field $B_{f}$ from initial field $B_{0}$ of either zero or a saturating field $B_{\mathrm{MAX}}=2 J_{1}$, the system is annealed [via $\Gamma(t)$ and $\left.J_{1}(t)\right]$ under $B_{0} ; B(t)$ is swept at the end of the protocol from $B_{0}$ to $B_{f}$ at a rate of $d t / d\left(B / B_{\mathrm{MAX}}\right)=1 \mu \mathrm{s}, 10 \mu \mathrm{s}$, or $100 \mu \mathrm{s}$ before $J(t)$ and $\Gamma(t)$ are rapidly quenched prior to state readout $\left[B_{f}=1, \frac{3}{2}\right.$, and 2 shown for $\left.d t / d\left(B / B_{\mathrm{MAX}}\right)=100 \mu \mathrm{s}\right]$. (b) Magnetization curves. Shown are measurements at $T=10 \mathrm{mK}$ for increasing field $\left(B_{0}=0\right.$, left) and decreasing field $\left(B_{0}=2\right.$, right) with a low and high transverse field (top and bottom, respectively). The black curves show equilibrium estimates (Appendix A). (c) The FIM long-range order $m_{\mathrm{FIM}}$, which is saturated in the threefold degenerate ground state of the 1/3 plateau. 
evolution controlled by $\mathcal{H}$ can reproduce the three steps. Near-term QA processors will be able to simulate more flexible geometries [35], including longer spin chains.

The structure of output states can shed light on the effect of quantum fluctuations at equilibrium and out of equilibrium. Figure 5 shows representative output states from the QA simulation. Figures 5(a) and 5(b) show equilibrium states at $B / J_{1}=1.48$ for $\Gamma / J_{1}=0.51$ and $\Gamma / J_{1}=1.06$, respectively. We see clear evidence of the role of the transverse field in suppressing FIM LRO while remaining in the $\mathcal{S}$ manifold. In Figs. 5(c) and 5(d), we see out-ofequilibrium states for the fastest sweep of $B / J_{1}$ from 2 to 1 . In this case, we see far more FIM LRO in the high$\Gamma$ case [in line with Fig. 4(c)] and many competing FIM domains in the low- $\Gamma$ case. At the domain boundaries, we see a variety of trapped defects, including up-down-down plaquettes and broken spin chains with nearly zero average magnetization, which contribute to the overshooting of the 1/3 plateau seen in Fig. 4(b). The presence of broken-chain defects indicates that the out-of-equilibrium dynamics is not fully captured by the low-energy effective model, in which spin chains are treated as single spins [33].

Quantum fluctuations can smooth the hysteresis of the system in two ways: first, by hastening spin-flip dynamics in general; and, second, by broadening the phase transition at $B_{c}$ with the intermediate shoulder seen in Fig. 3. In Appendix C, we present path-integral Monte Carlo (PIMC) simulations, which show that the acceleration is much more significant when single-spin updates are employed than when spin chains are updated collectively. Despite the fact that PIMC does not accurately simulate QA dynamics $[15,36,37]$, this suggests that the acceleration is a matter of increased qubit dynamics, rather than being reflective of a new path through the phase transition that avoids metastable configurations (see Appendix C).

\section{CONCLUSIONS}

We demonstrated a large-scale out-of-equilibrium simulation of a frustrated transverse field Ising model using a programmable superconducting QA processor. When quantum fluctuations are small, we observe the signature metastable magnetization curves also seen in experimental measurements of spin-chain compounds. The introduction of larger quantum fluctuations produces both dynamical and equilibrium effects on the system. Out-of-equilibrium simulations are a promising application for quantum simulation: standard quantum Monte Carlo methods - be they single-spin Glauber dynamics or imaginary-time cluster update dynamics (e.g., Swendsen-Wang) - cannot faithfully reproduce the real-time evolution of a quantum system [15,36,38]. Although the evolution of a quantum system in the presence of a thermal bath is fundamental to QA, the relationship between the QA dynamics and the time evolution of a quantum Ising model has not been established experimentally in detail for large systems. Moreover, the spin-chain system that we simulate is only qualitatively similar to real compounds - future hardware generations with greater control and more flexible Hamiltonian terms and geometry [35,39] will allow deeper and more realistic simulations of this nature.

Although independent control of transverse and longitudinal magnetic fields is difficult to achieve for in situ crystal studies, a laboratory reproduction of our findings in $\mathrm{Ca}_{3} \mathrm{Co}_{2} \mathrm{O}_{6}$ or other easy-axis magnets with similar triangular chain structure would be of great interest. This would mark the first example of a quantum simulation preceding confirmation in a physical sample, an important milestone in quantum simulation for materials science.

\section{ACKNOWLEDGMENTS}

We gratefully acknowledge the contributions of the teams at D-Wave, who designed, fabricated, and calibrated the QA processor used in this work. We thank Vivien Zapf, Arnab Banerjee, and Paul Kairys for helpful conversations.

\section{APPENDIX A: METHODS}

\section{Experimental methods}

The QA processor used is a D-Wave 2000Q system, in which 2041 of 2048 superconducting radio-frequency superconducting quantum interference device (rf-SQUID) flux qubits are operable. Effective qubit temperatures of between $10 \mathrm{mK}$ and $24 \mathrm{mK}$ are measured via qubit susceptibility, following the methods of [12] (SM page 8). The effective TFIM parameters $\Gamma$ and $J_{1}$ are extracted using a combination of spectroscopy measurements and diagonalization of the Hamiltonian of 12 qubits arranged in a representative gadget, as detailed in the "Methods" section of Ref. [15] for a different superconducting processor of similar design. Similarly, the leading qubit-to-spin nonideality of background susceptibility is compensated in the coupling energies as in the "Methods" section of Ref. [15].

In this work, we study a single lattice programmed into the qubit architecture; this has the same 1800-qubit size as studied in Ref. [14] but due to inoperable qubits only uses 1764 spins. Longitudinal fields $H=B / J_{1}$ can be programmed either negative or positive; we run experiments for $H=0,0.04, \ldots, 2$ in both directions and combine symmetric results.

With each programming of the quantum processing unit (QPU), we draw 100 samples. The out-of-equilibrium experiments are performed using the protocol described in the main text and shown in Fig. 4; these protocols require the " $H$-gain" control. The equilibrium estimates are generated using QEMC [14,15,31], a method that, analogous to Markov-chain Monte Carlo, forms a Monte Carlo chain of reverse-annealing protocols that dwell at the desired 

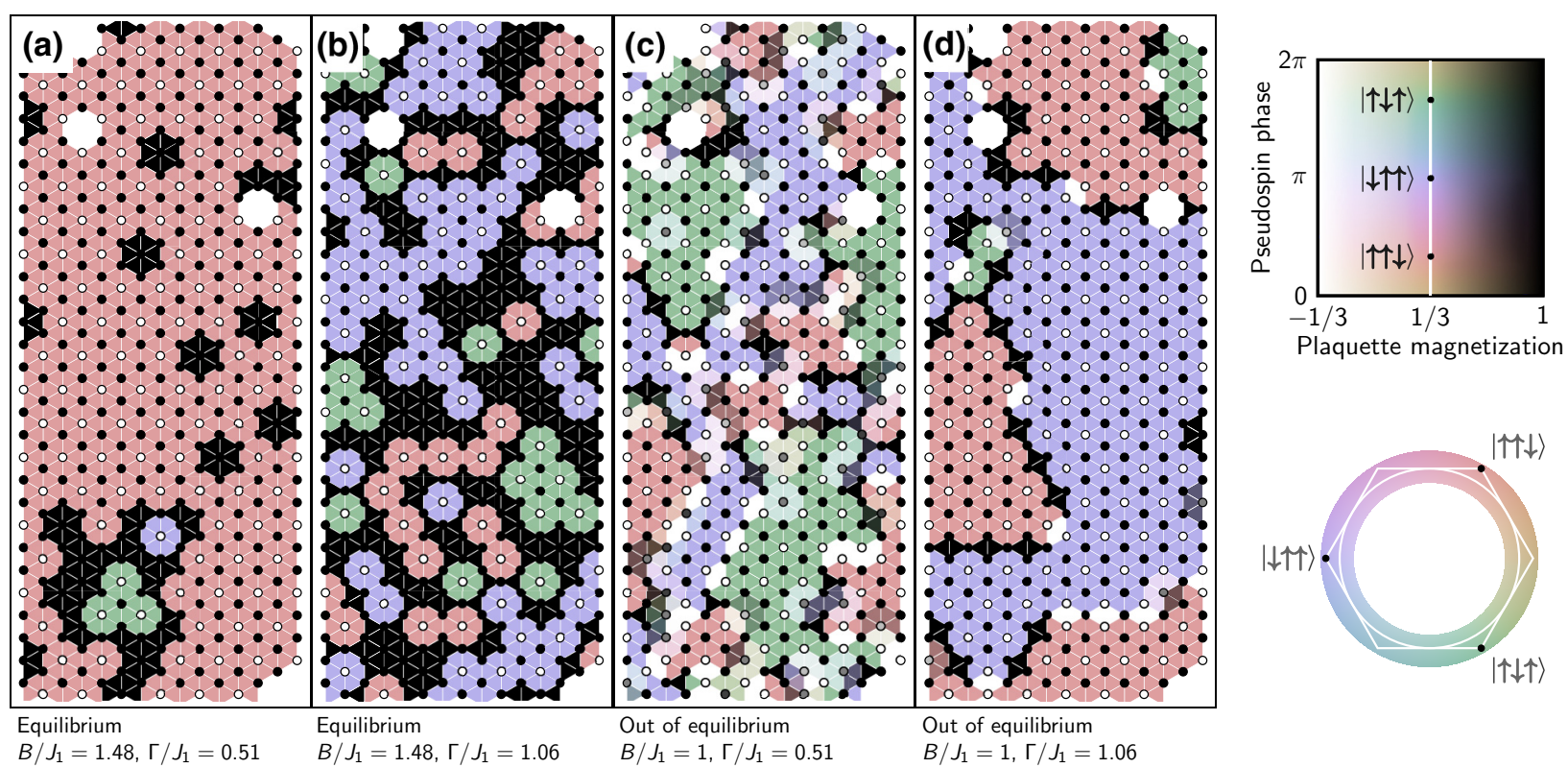

FIG. 5. Experimental output samples. The darkness of the markers indicates magnetization of the four-qubit chain; the darkness of the plaquettes indicates plaquette magnetization. The plaquette hue indicates the pseudospin phase; the $1 / 3$ plateau has three degenerate FIM ground states with varying phase. The lattice is periodic at the top (bottom) and open on the left (right). Six of 450 sites are empty due to inoperable qubits. (a),(b) Equilibrium samples at $H=1.48$; the transverse field modulates long-range order: (a) at $\Gamma / J_{1}=0.51$, the system adopts long-range FIM order and is dominated by plaquettes of a single phase, with $m_{\mathrm{FIM}}$ large; (b) with $\Gamma / J_{1}=1.06$, the system is characterized by competing FIM domains, with saturated plaquettes at the interfaces. (c),(d) Out-of-equilibrium samples, with $H$ decreasing from 2 to 1 over $0.5 \mu \mathrm{s}$ : (c) with $\Gamma / J_{1}=0.51$, the system is chaotic, with many small FIM domains - the interfaces of these domains host trapped excitations, many in the form of broken four-qubit chains with magnetization zero; $(\mathrm{d})$ with $\Gamma / J_{1}=1.06$, the system forms large FIM domains during the sweep of $H$ - the interfaces between these domains host plaquettes of magnetization +1 and $-1 / 3$.

Hamiltonian rather than annealing to it. The QEMC (equilibrium estimating) calls also draw 100 samples, of which we discard the first 50 as QEMC burn-in. We dwell for $500 \mu$ s per step, except in the case where $\Gamma / J_{1}<0.77$, where relaxation is slow. In this case, we reverse anneal to an intermediate value of $\Gamma / J_{1}$, then anneal "forward" (i.e., attenuating $\Gamma$ ) to the target value of $\Gamma / J_{1}$ over $250 \mu \mathrm{s}$, then dwell at the target $\Gamma / J_{1}$ for the remaining $250 \mu \mathrm{s}$. Figure 6 shows the waveforms for a single reverse-annealing step in each of these cases.

In practice, the relative strengths of $\Gamma$ and $J_{1}$ are not controlled independently but, rather, through the single annealing parameter $s$; the functions $\Gamma(s)$ and $J_{1}(s)$ are fixed functions of the QA processor, which allows timedependent control of $s(t)$ (cf. Ref. [15], "Methods"). Varying $\Gamma$ for fixed $J_{1}$ therefore requires tuning $\Gamma(s)$ and $J_{1}(s)$ via $s$ and then compensating by adjusting the individual programmed coupling terms.

All reported data are taken over 120 individual programmings, representing 12000 samples for out of equilibrium and 6000 samples for equilibrium. The reported bulk averages (magnetization, susceptibility, and FIM order) are taken over nonboundary spins to reduce the effect of open boundaries and defects.
All QA methods used in this study are available to external users of D-Wave systems, with the exception of changing temperature.

\section{Calibration refinement}

QA simulations in highly degenerate spaces have shown the importance of fine tuning the Hamiltonian parameters calibration to compensate for device variation and boundary conditions [14,15,31,40] (specifically cf. Ref. [14], "Extended Data," Fig. 6). The idea of per-spin tuning of longitudinal fields in the presence of open boundaries goes back to the 1970s [30] and although it now seems obsolete for computer simulations, here it allows us to better simulate the thermodynamic limit of a large system in a uniform magnetic field [31], in spite of the fact that limited qubit connectivity prevents us from implementing fully periodic boundaries.

In contrast to previous studies on cylindrical lattices $[14,15]$ that attempted to faithfully simulate a finite system with partially open boundaries, here we are interested in the thermodynamic limit. We therefore use the same approach of iteratively tuning $J_{i j}$ terms to homogenize spin-spin correlations. However, instead of doing 

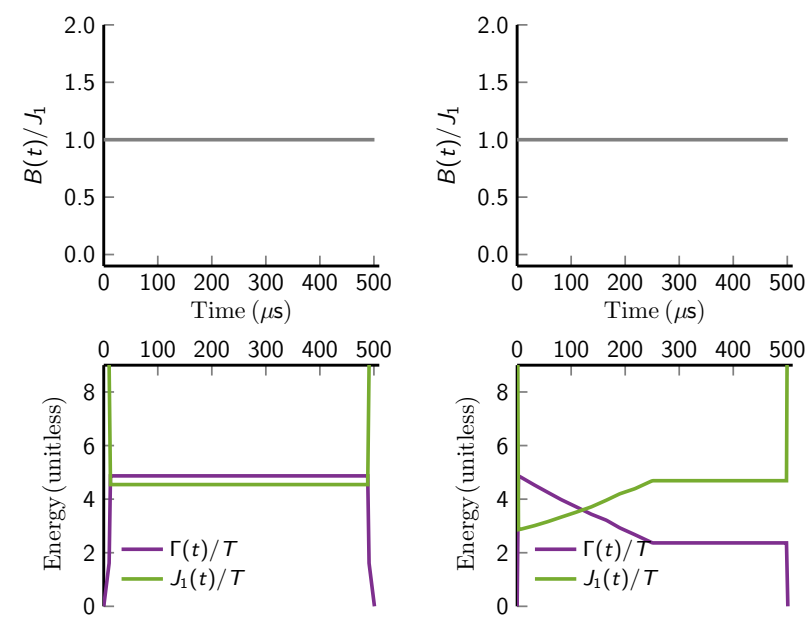

FIG. 6. Reverse-annealing protocols for equilibrium estimates. For estimating the equilibrium statistics, we perform "quantum evolution Monte Carlo," forming a chain of reverse anneals. Each reverse anneal starts from a classical state with $\Gamma=0$, then increases $\Gamma$, dwells, and decreases $\Gamma$ to 0 , arriving at a new classical state. In the easy case (left), the protocol involves a simple dwell. In the slow-relaxing case (right), $\Gamma$ is attenuated from a higher value before the dwell. Each chain repeats the reverse anneal 100 times, producing 100 classical output samples that ideally represent projections of the system to the $\sigma^{z}$ basis. In both cases, $B(t) / J_{1}$ is held constant [e.g., $B(t) / J_{1}=1$ as shown here], in contrast to the out-of-equilibrium protocols shown in Fig. 4.

this over small rotational symmetry groups of the cylinder, we homogenize correlations and magnetizations across the entire lattice.

When homogenizing the coupling terms, we tune terms using $H=B / J_{1}=0$ observations and propagate the couplings to all simulated $H$ values. When homogenizing the per-spin field strengths, we use all observations and employ smoothing of the Hamiltonian terms so that relative field strengths vary smoothly as a function of $H$.

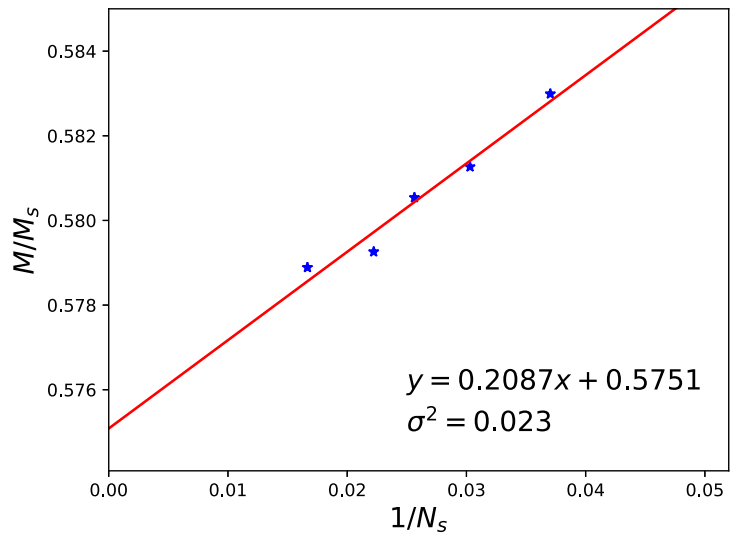

FIG. 8. The finite-size scaling of the magnetization that maximizes the entropy within the subspace $\mathcal{S}$ of low-energy states.

\section{APPENDIX B: ENTROPIC ORIGIN OF MAGNETIZATION SHOULDER}

Further evidence for the entropic origin of the shoulder is shown in Fig. 7, where we compute the average local degeneracy of observed states, defined as the number of states reachable from a given state by moving a single down-spin chain to a neighboring site. This local entropy is found to peak at the phase transition, with a peak height that increases with both quantum and thermal fluctuations, in the form of changing $\Gamma$ and $T$, respectively.

As explained in the main text, $\tilde{\mathcal{H}}(\tilde{\Gamma}=0)$ has an extensive ground-state degeneracy at the effective saturation field $\widetilde{B}_{\text {sat }}=4 B_{\text {sat }}=6 J$, which is lifted by the transverse field. The ground space $\mathcal{S}$ is generated by spin states $\left|\phi_{j}\right\rangle$ that do not contain any pair of nearest-neighbor spins antialigned with the longitudinal field $B$. To compute the magnetization value that maximizes the number of states (i.e., the entropy) within this low-energy sector, we randomly select spin states that satisfy the above-mentioned constraint by starting from the fully polarized state and flipping the spin of each site with probability $1 / 2$ if none
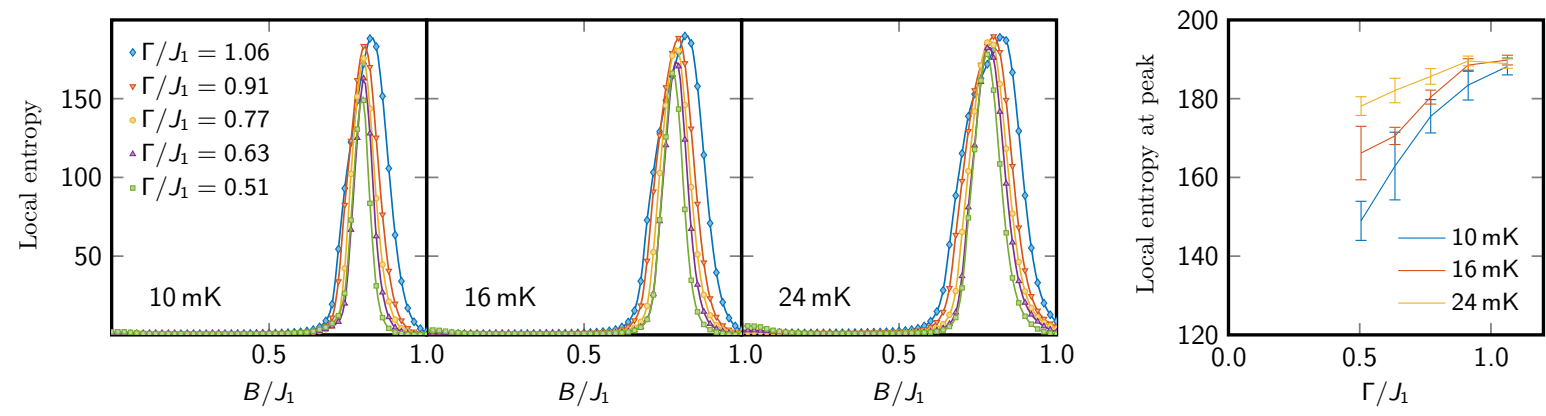

FIG. 7. The local entropy of the observed states. The ground state at $\Gamma=0, T=0, H=\frac{3}{2}$ has extensive entropy, consisting of triangular plaquettes with one or zero down-spins, which is lifted by the transverse field. The local entropy, which we define as the number of neighboring states differing by a swap of a single up-down spin chain pair, becomes increasingly favorable with increasing $\Gamma$ and $T$. The error bars indicate the standard deviation of the estimates for individual 50-sample QA programmings. 
of the nearest neighbors has already been flipped. This procedure is implemented on a finite triangular lattice with periodic boundary conditions. For each lattice size $N_{s}=27,33,39,45,60$, we compute a magnetization histogram and extract the "optimal" magnetization value that maximizes the number of states. Since the magnetization takes discrete values on a finite lattice, the number of samples is increased until the "optimal" magnetization stops fluctuating. The resulting optimal magnetization value as a function of the inverse of the system size is shown in Fig. 8, along with a linear extrapolation to the thermodynamic limit (infinite system size $N_{s} \rightarrow \infty$ ). The magnetization value that results from this extrapolation is $M_{m} / M_{s} \sim 0.575$.

\section{APPENDIX C: QMC SIMULATIONS}

Path-integral Monte Carlo is a common and accurate tool for estimating equilibrium statistics of TFIM systems at finite temperature. Although in some incoherent

\section{QMC, single-spin updates}

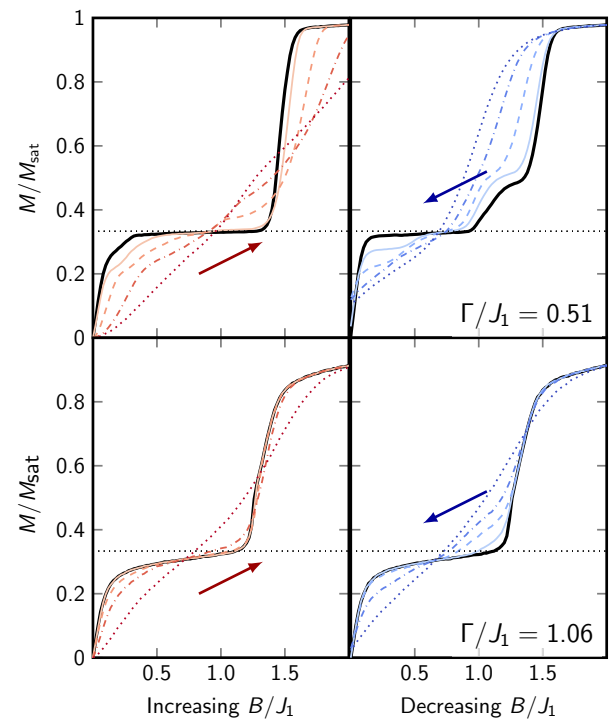

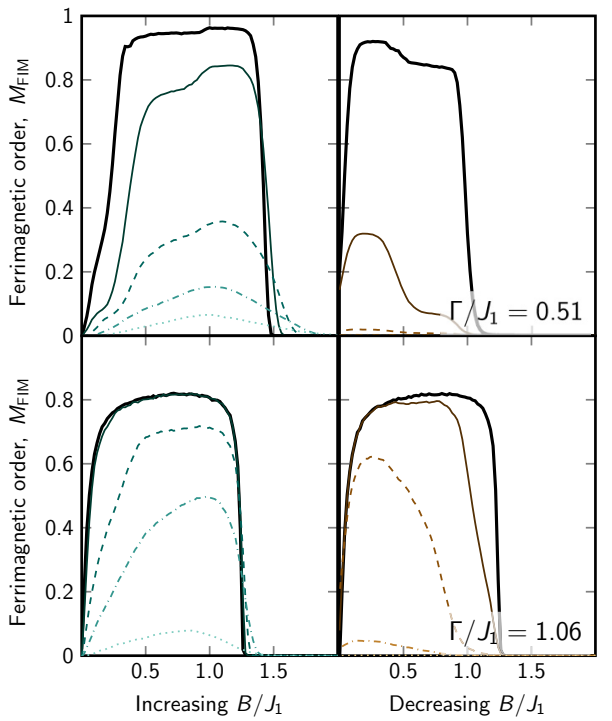

QMC, spin-chain updates

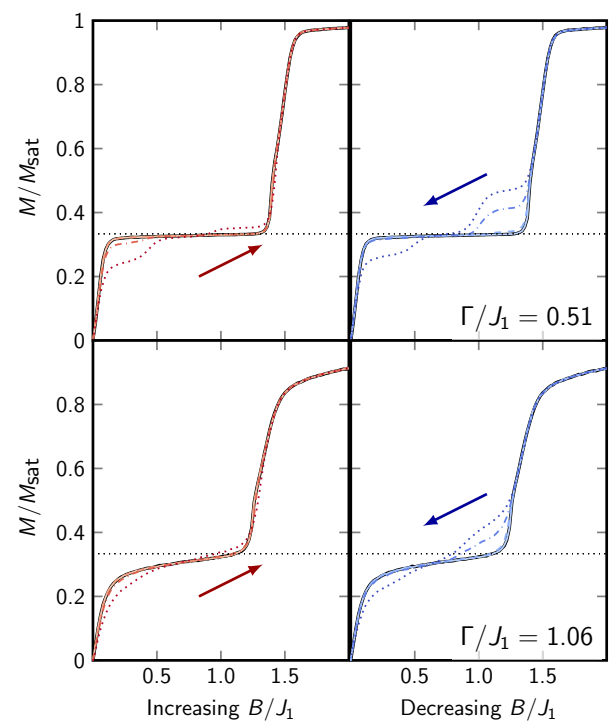

$\begin{array}{ll}-10^{7} \text { sweeps } & -10^{7} \text { sweeps } \\ -10^{6} \text { sweeps } & 10^{6} \text { sweeps } \\ -\cdots 10^{5} \text { sweeps } & -\cdots 10^{5} \text { sweeps } \\ -\cdots 10^{4} \text { sweeps } & -\cdots 10^{4} \text { sweeps } \\ \cdots \cdots 10^{3} \text { sweeps } & \cdots \cdots 10^{3} \text { sweeps }\end{array}$

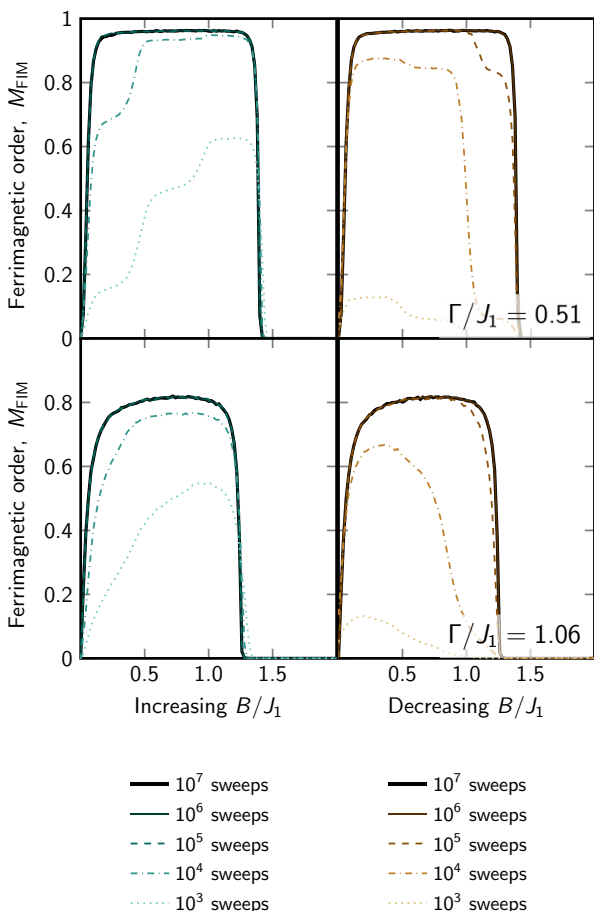

FIG. 9. Out-of-equilibrium QMC simulations. The simulations are performed on a fully periodic lattice with no site vacancies, with a longitudinal field swept from $10^{3}$ to $10^{7}$ Monte Carlo sweeps. Sweeps are performed either one spin at a time (top) or four spins at a time (bottom). Compare with Fig. 4. 
cases the dynamics can be directly mapped to tunneling in a quantum system [41], this is not true in general [36,37]. In particular, PIMC fails to reproduce QA relaxation dynamics in this spin-chain lattice in the absence of a longitudinal field [15]. Therefore QA, which involves relaxation of an engineered quantum system, may eventually provide more faithful simulation of the relaxation dynamics of effective transverse-field Ising models such as $\mathrm{TmMgGaO}$.

Here, we compare and contrast the out-of-equilibrium QA results with qualitatively analogous QMC simulations. Figure 9 shows results on a 1764-spin lattice $(21 \times 21$ four-spin chains) with fully periodic boundary conditions (note that the current QA qubit connectivity does not allow for fully periodic boundaries in this lattice). We consider two QMC variants: one that uses single-spin updates and one that collectively updates the four-spin chains. Both are detailed in the Supplemental Material of Ref. [15]. We emphasize that these QMC experiments are on a lattice with different boundary conditions than the QA experiments. Consequently, the fine tuning that we performed in QA to better simulate the thermodynamic limit is not applicable to the PIMC case. Thus these results are only intended to provide qualitative comparison. Unlike Ref. [15], we are not attempting a quantitative comparison of relaxation dynamics.

Most obviously, and particularly for large $\Gamma$, the QMC results differ from QA in that they do not saturate either the FIM LRO or the magnetization. This is because of canting in the $\sigma^{x}$ direction due to the transverse field; this canting is destroyed as a local relaxation during the QA readout quench. This relaxation - and the corresponding failure of QA to reproduce accurate statistics when quenching from a regime of fast dynamics - has been observed both in frustrated systems [14] and isolated chains [42]. In one sense, this is a drawback of the current QA methods, albeit one that is easily recovered with postprocessing (see [15], Supplemental Fig. 18). A preferable alternative would be the development of a faster quench in QA, which can faithfully project to the $\sigma^{z}$ basis without distortion. In another sense, however, the method of periodically driving toward a classical ground state is a potentially powerful computational method. This would be similar in nature to proposed algorithmic manipulation of energy splitting caused by the transverse field, wherein a potentially undesirable quality of quantum annealing [43] is exploited for computational gain [44].

We note that with spin-chain updates, the larger transverse field has little effect on the hysteresis; this is not surprising since the spin-chain updates effectively reduce the system to a $2 \mathrm{D}$ triangular AFM. With single-spin updates, the transverse field indeed accelerates the generation of FIM long-range order as it does in QA. This is expected, since the origin of the four-spin update is not physical but, rather, algorithmic. We expect that future experiments on systems with longer spin chains will elucidate the comparison between $\mathrm{QMC}$ and $\mathrm{QA}$ in this context.

[1] V. Hardy, D. Flahaut, M. R. Lees, and O. A. Petrenko, Magnetic quantum tunneling in $\mathrm{Ca}_{3} \mathrm{Co}_{2} \mathrm{O}_{6}$ studied by ac susceptibility: Temperature and magnetic-field dependence of the spin-relaxation time, Phys. Rev. B 70, 1 (2004).

[2] J. W. Kim, E. D. Mun, X. Ding, A. Hansen, M. Jaime, N. Harrison, H. T. Yi, Y. Chai, Y. Sun, S. W. Cheong, and V. S. Zapf, Metastable states in the frustrated triangular compounds $\mathrm{Ca}_{3} \mathrm{Co}_{2-x} \mathrm{Mn}_{x} \mathrm{O}_{6}$ and $\mathrm{Ca}_{3} \mathrm{Co}_{2} \mathrm{O}_{6}$, Phys. Rev. B 98, 1 (2018).

[3] S. Agrestini, L. C. Chapon, A. Daoud-Aladine, J. Schefer, A. Gukasov, C. Mazzoli, M. R. Lees, and O. A. Petrenko, Nature of the Magnetic Order in $\mathrm{Ca}_{3} \mathrm{Co}_{2} \mathrm{O}_{6}$, Phys. Rev. Lett. 101, 2 (2008).

[4] J. G. Cheng, J. S. Zhou, and J. B. Goodenough, Thermal conductivity, electron transport, and magnetic properties of single-crystal $\mathrm{Ca}_{3} \mathrm{Co}_{2} \mathrm{O}_{6}$, Phys. Rev. B-Condens. Matter Mater. Phys. 79, 1 (2009).

[5] C. L. Fleck, M. R. Lees, S. Agrestini, G. J. McIntyre, and O. A. Petrenko, Field-driven magnetisation steps in $\mathrm{Ca}_{3} \mathrm{Co}_{2} \mathrm{O}_{6}$ : A single-crystal neutron-diffraction study, EPL (Europhys. Lett.) 90, 67006 (2010).

[6] Y. Kamiya and C. D. Batista, Formation of Magnetic Microphases in $\mathrm{Ca}_{3} \mathrm{Co}_{2} \mathrm{O}_{6}$, Phys. Rev. Lett. 109, 2 (2012).

[7] Y. B. Kudasov, A. S. Korshunov, V. N. Pavlov, and D. A. Maslov, Relaxation and magnetization dynamics of frustrated spin-chain systems, J. Low Temp. Phys. 159, 76 (2010).

[8] Y. B. Kudasov, A. S. Korshunov, V. N. Pavlov, and D. A. Maslov, Dynamics of magnetization in frustrated spin-chain system $\mathrm{Ca}_{3} \mathrm{Co}_{2} \mathrm{O}_{6}$, Phys. Rev. B 78, 132407 (2008).

[9] R. Soto, G. Martínez, M. N. Baibich, J. M. Florez, and P. Vargas, Metastable states in the triangular-lattice Ising model studied by Monte Carlo simulations: Application to the spin-chain compound $\mathrm{Ca}_{3} \mathrm{Co}_{2} \mathrm{O}_{6}$, Phys. Rev. B-Condens. Matter Mater. Phys. 79, 1 (2009).

[10] A. Maignan, V. Hardy, S. Hébert, M. Drillon, M. R. Lees, O. Petrenko, D. M. K. Paul, and D. Khomskii, Quantum tunneling of the magnetization in the Ising chain compound $\mathrm{Ca}_{3} \mathrm{Co}_{2} \mathrm{O}_{6}$, J. Mater. Chem. 14, 1231 (2004).

[11] J. Brooke, D. Bitko, T. F. Rosenbaum, and G. Aeppli, Quantum annealing of a disordered magnet, Science 284, 779 LP (1999).

[12] M. W. Johnson, et al., Quantum annealing with manufactured spins, Nature 473, 194 (2011).

[13] R. Harris, et al., Phase transitions in a programmable spin glass simulator, Science 165, 162 (2018).

[14] A. D. King, et al., Observation of topological phenomena in a programmable lattice of 1,800 qubits, Nature 560, 456 (2018).

[15] A. D. King, et al., Scaling advantage over path-integral Monte Carlo in quantum simulation of geometrically frustrated magnets, Nat. Commun. 12, 1113 (2021).

[16] P. Weinberg, M. Tylutki, J. M. Rönkkö, J. Westerholm, J. A. Åström, P. Manninen, P. Törmä, and A. W. Sandvik, 
Scaling and Diabatic Effects in Quantum Annealing with a D-Wave Device, Phys. Rev. Lett. 124, 090502 (2020).

[17] S. Zhou, D. Green, E. D. Dahl, and C. Chamon, Experimental realization of spin liquids in a programmable quantum device, ArXiv:2009.07853 (2020).

[18] S. V. Isakov and R. Moessner, Interplay of quantum and thermal fluctuations in a frustrated magnet, Phys. Rev. B 68, 104409 (2003).

[19] H. Kageyama, K. Yoshimura, K. Kosuge, H. Mitamura, and T. Goto, Field-induced magnetic transitions in the onedimensional compound $\mathrm{Ca}_{3} \mathrm{Co}_{2} \mathrm{O}_{6}$, J. Phys. Soc. Jpn. 66, 1607 (1997).

[20] H. Kageyama, K. Yoshimura, K. Kosuge, M. Azuma, M. Takano, H. Mitamura, and T. Goto, Magnetic anisotropy of $\mathrm{Ca}_{3} \mathrm{Co}_{2} \mathrm{O}_{6}$ with ferromagnetic Ising chains, J. Phys. Soc. Jpn. 66, 3996 (1997).

[21] A. Maignan, C. Michel, A. C. Masset, C. Martin, and B. Raveau, Single crystal study of the one dimensional $\mathrm{Ca}_{3} \mathrm{Co}_{2} \mathrm{O}_{6}$ compound: Five stable configurations for the Ising triangular lattice, Eur. Phys. J. B 15, 657 (2000).

[22] V. Hardy, M. R. Lees, O. A. Petrenko, D. M. Paul, D. Flahaut, S. Hébert, and A. Maignan, Temperature and time dependence of the field-driven magnetization steps in $\mathrm{Ca}_{3} \mathrm{Co}_{2} \mathrm{O}_{6}$ single crystals, Phys. Rev. B 70, 064424 (2004).

[23] T. Moyoshi and K. Motoya, Incommensurate magnetic structure and its long-time variation in a geometrically frustrated magnet $\mathrm{Ca}_{3} \mathrm{Co}_{2} \mathrm{O}_{6}$, J. Phys. Soc. Jpn. 80, 034701 (2011).

[24] F. A. Cevallos, K. Stolze, T. Kong, and R. Cava, Anisotropic magnetic properties of the triangular plane lattice material $\mathrm{TmMgGaO}_{4}$, Mater. Res. Bull. 105, 154 (2018).

[25] H. Li, Y. D. Liao, B.-B. Chen, X.-T. Zeng, X.-L. Sheng, Y. Qi, Z. Y. Meng, and W. Li, Kosterlitz-Thouless melting of magnetic order in the triangular quantum Ising material $\mathrm{TmMgGaO}_{4}$, Nat. Commun. 11, 1111 (2020).

[26] Y. Shen, C. Liu, Y. Qin, S. Shen, Y.-D. Li, R. Bewley, A. Schneidewind, G. Chen, and J. Zhao, Intertwined dipolar and multipolar order in the triangular-lattice magnet $\mathrm{TmMgGaO}_{4}$, Nat. Commun. 10, 4530 (2019).

[27] C. Liu, C.-J. Huang, and G. Chen, Intrinsic quantum Ising model on a triangular lattice magnet $\mathrm{TmMgGaO}_{4}$, Phys. Rev. Res. 2, 043013 (2020).

[28] Y. Li, S. Bachus, H. Deng, W. Schmidt, H. Thoma, V. Hutanu, Y. Tokiwa, A. A. Tsirlin, and P. Gegenwart, Partial Up-Up-Down Order with the Continuously Distributed Order Parameter in the Triangular Antiferromagnet $\mathrm{TmMgGaO}_{4}$, Phys. Rev. X 10, 011007 (2020).

[29] Y. Da Liao, H. Li, Z. Yan, H.-T. Wei, W. Li, Y. Qi, and Z. Y. Meng, Phase diagram of the quantum Ising model on a triangular lattice under external field, Phys. Rev. B 103, 104416 (2021).

[30] H. Müller-Krumbhaar and K. Binder, A "self consistent" Monte Carlo method for the Heisenberg ferromagnet, Z. Phys. A: Hadrons Nucl. 254, 269 (1972).

[31] P. Kairys, A. D. King, I. Ozfidan, K. Boothby, J. Raymond, A. Banerjee, and T. S. Humble, Simulating the ShastrySutherland Ising model using quantum annealing, PRX Quantum 1, 020320 (2020).

[32] R. Moessner and S. L. Sondhi, Ising models of quantum frustration, Phys. Rev. B 63, 224401 (2001).

[33] Y. B. Kudasov, Steplike Magnetization in a Spin-Chain System: $\mathrm{Ca}_{3} \mathrm{Co}_{2} \mathrm{O}_{6}$, Phys. Rev. Lett. 96, 027212 (2006).

[34] A. Maignan, C. Michel, A. C. Masset, C. Martin, and B. Raveau, Single crystal study of the one dimensional $\mathrm{Ca}_{3} \mathrm{Co}_{2} \mathrm{O}_{6}$ compound: Five stable configurations for the Ising triangular lattice, Eur. Phys. J. B 15, 657 (2000).

[35] K. Boothby, P. Bunyk, J. Raymond, and A. Roy, Nextgeneration topology of D-Wave quantum processors, ArXiv:2003.00133 (2020).

[36] E. Andriyash and M. H. Amin, Can quantum Monte Carlo simulate quantum annealing? ArXiv:1703.09277 (2017).

[37] Y. Bando and H. Nishimori, Simulated quantum annealing as a simulator of non-equilibrium quantum dynamics, ArXiv:2106.00928 (2021).

[38] K. Kechedzhi, V. Smelyanskiy, J. R. McClean, V. S. Denchev, M. Mohseni, S. Isakov, S. Boixo, B. Altshuler, and H. Neven, in 13th Conference on the Theory of Quantum Computation, Communication and Cryptography (TQC 2018), Leibniz International Proceedings in Informatics (LIPIcs), Vol. 111, edited by S. Jeffery (Schloss Dagstuhl-Leibniz-Zentrum fuer Informatik, Dagstuhl, Germany, 2018), p. 9:1.

[39] I. Ozfidan, et al., Demonstration of a Nonstoquastic Hamiltonian in Coupled Superconducting Flux Qubits, Phys. Rev. Appl. 13, 034037 (2020).

[40] K. Nishimura, H. Nishimori, and H. G. Katzgraber, Griffiths-McCoy singularity on the diluted Chimera graph: Monte Carlo simulations and experiments on quantum hardware, Phys. Rev. A 102, 042403 (2020).

[41] S. V. Isakov, G. Mazzola, V. N. Smelyanskiy, Z. Jiang, S. Boixo, H. Neven, and M. Troyer, Understanding Quantum Tunneling through Quantum Monte Carlo Simulations, Phys. Rev. Lett. 117, 180402 (2016).

[42] Z. G. Izquierdo, T. Albash, and I. Hen, Testing a quantum annealer as a quantum thermal sampler, ArXiv:2003.00361 (2020).

[43] S. Boixo, T. Albash, F. M. Spedalieri, N. Chancellor, and D. A. Lidar, Experimental signature of programmable quantum annealing, Nat. Commun. 4, 2067 (2013).

[44] N. Chancellor, Fluctuation-guided search in quantum annealing, Phys. Rev. A 102, 62606 (2020). 